\title{
Para-Hydrogen Narrow Filament Evaporation at Low Temperature
}

\author{
T. G. Elizarova ${ }^{1}$, A. A. Gogolin ${ }^{2}$, and S. Montero ${ }^{3}$ \\ ${ }^{1}$ M. V. Keldysh Institute of Applied Mathematics, Russian Academy of Science \\ Miusskaya sq. 4,125046, Moscow (Russia) \\ ${ }^{2}$ L. D. Landau Institute of Theoretical Physics, Russian Academy of Science \\ Vorobiovskoe sh. 2, 117332, Moscow (Russia) \\ ${ }^{3}$ Laboratory of Molecular Fluid Dynamics@ Instituto de Estructura de la Materia, CSIC \\ Serrano 121, 28006, Madrid (Spain)
}

\begin{abstract}
Undercooling of liquid para-hydrogen $\left(\mathrm{pH}_{2}\right)$ below its freezing point at equilibrium $(13.8 \mathrm{~K})$ has been shown recently in flowing micro-filaments evaporating in low density background gas [M. Kühnel et al, Phys. Rev. Lett. 106, 245301 (2011)]. An hydrodynamical model accounting for this process is reported here. Analytical expressions for the local temperature $T$ of a filament, averaged over its cross section, are obtained as a function of distance $z$ to the nozzle. Comparison with the experiment is shown. It is shown also that the thermocapillary forces induce a parabolic profile of velocity across the jet.
\end{abstract}

Keywords: Evaporative supercooling, Hydrogen filaments, Hydrodynamics, Modeling

PACS: $64.70 . \mathrm{fm}, 64.60 . \mathrm{Ht}, 67.63 .-\mathrm{r}$

\section{INTRODUCTION}

Recent experimental results on super-cooled liquid $\mathrm{pH}_{2}$ filaments [1] enable studying in detail the process of crystallization in these systems at low temperatures $(9<T<17 \mathrm{~K})$. The problem is connected with the conjectured superfluidity in $\mathrm{pH}_{2}$ at low temperatures [2] - [4]

In order to study in detail the space structure of the filaments we propose a simple theoretical model for the balance of the heat flows between the filament and the rarefied background gas. The temperature distribution along the filament is predicted in reasonable agreement with experiment. For a filament of diameter $D=2 R_{0}=4 \mu \mathrm{m}$ and a jet of velocity $u_{0}=200 \mathrm{~m} / \mathrm{s}$ we get a maximum length scale of $L=1.1 \mathrm{~mm}$, also in agreement with the experiment [1].

The exact analytical results for the temperature gradient along the filament make it possible to study the effects of surface-tension on the velocity profile inside the narrow flow. Although this disturbance in the velocity profile is small, it might induce crystal growth habits differing from those of bulk solid $\mathrm{pH}_{2}$.

\section{HEAT BALANCE EQUATION}

The length of the liquid filament is determined by the usual heat balance equations for the main thermal flows. As a result the decrease of temperature $T$ along the stream length $z$ is related with the thermal flow $Q_{1}$ by

$$
d Q_{1}=u_{0} \rho_{0} c_{1} d T \frac{\pi}{4} D^{2},
$$

where $u_{0}$ is the stream velocity, $\rho_{0}$ is the liquid hydrogen density, $c_{1}$ is the liquid hydrogen heat capacity and $D$ is the stream diameter. This decrease is equal to the corresponding heat flux due to vaporization

$$
d Q_{2}=\pi D d z \rho(T) c_{2} T \bar{v}_{\perp \rho} .
$$

Here $c_{2}=5 R / 2$ is the heat capacity for the $\mathrm{pH}_{2}$ vapor with negligible contribution from rotation due to the very low temperature, $\rho(T)$ is the density of the vapor, and $\bar{v}_{\perp \rho}=\sqrt{\pi k T /(2 m)}$ is the average radial velocity. $R$ is the gas constant, $k$ is Boltzmann constant and $m$ the mass of $\mathrm{H}_{2}$ molecule.

28th International Symposium on Rarefied Gas Dynamics 2012

AIP Conf. Proc. 1501, 1236-1241 (2012); doi: 10.1063/1.4769683

(C) 2012 American Institute of Physics 978-0-7354-1115-9/\$30.00 
The dependence of density on temperature

$$
\rho(T)=\rho_{0} \exp \left(-\frac{q}{R T}\right)
$$

is described in terms of the usual evaporation heat $q$ [5].

Due to the linear dependence for $c_{1}(T)=A_{2} T[6,7]$, combining Eqs. (1), (2) and (3) leads to

$$
z(T)=\frac{D}{5 \sqrt{\pi}} u \frac{A_{2}}{R} \sqrt{\frac{m}{k}} \int_{T}^{T_{0}} \frac{d \tau}{\sqrt{\tau}} \exp \left(\frac{q}{R \tau}\right),
$$

where $T_{0}=17 \mathrm{~K}$ is the initial temperature of the liquid hydrogen in the stream.

From Eq. (4) we get

$$
\frac{1}{D} z(T)=L_{0} B(T)\left(\frac{T}{T_{1}}\right)^{3 / 2} \exp \left(T_{1} / T\right), \quad T_{1}=\frac{q}{R},
$$

where

and

$$
L_{0}=\frac{1}{5 \sqrt{\pi}} u_{0} \frac{A_{2}}{R}(m q / k R)^{1 / 2}
$$

In the experiment [1]

$$
B(T)=\int_{0}^{\alpha} \frac{e^{-t} d t}{(1-\beta t)^{3 / 2}}, \quad \beta=\frac{R T}{q}, \quad \alpha=\frac{q}{R}\left(\frac{1}{T}-\frac{1}{T_{0}}\right) .
$$

$$
u_{0}=(200 \pm 20) \mathrm{m} / \mathrm{s}, \quad A_{2}=0.932 \frac{\mathrm{J}}{\mathrm{mol} \mathrm{K}^{2}}, \quad q=904 \frac{\mathrm{J}}{\mathrm{mol}}, \quad T_{1}=109 \mathrm{~K},
$$

which lead to the value of $L_{0}=0.391$.

For the temperature interval $9<T<T_{0}=17 \mathrm{~K}$ the small value of $\beta \sim 0.1<<1$ is obtained. The value of $\alpha \sim \beta^{-1} \sim 10>>1$ is large enough, so

$$
B(T) \approx 1+\frac{3 R T}{2 q}, \text { for } \quad T<0.7 T_{0}
$$

In the narrow interval $0.9 T_{0}<T<T_{0}$ the coefficient $B \rightarrow 0$ in a linear way:

$$
B(T) \approx \frac{T_{1}}{T_{0}}\left(1-\frac{T}{T_{0}}\right) \approx 6.42\left(1-\frac{T}{T_{0}}\right), \text { for } \quad 0.9 T_{0} \leq T \leq T_{0} .
$$

Equations (9) and (10) are thus the asymptotic form of $B(T)$ for two extremal cases. As a result the Eq. (5) together with Eqs. (9) and (10) give the simple analytical formula for $z(T)$. The computations for $B(T)$ and the resulting dependence $T(z)$ is shown in Figs. 1 and 2.

The entropy distribution along the filament can be obtained using the entropy equation

$$
T d S=c_{1} d T
$$

and the expression for the liquid hydrogen heat capacity $c_{1}=A_{2} T$. We immediately obtain

$$
S(z)=S_{0}+A_{2}\left(T(z)-T_{0}\right)
$$

where $S_{0}=S\left(T_{0}\right)$ is the entropy value at the nozzle exit. Because $T(z) \leq T_{0}$ and decreases along $z$, the entropy decreases along the filament.

The average temperature spread along a thin filament of liquid $\mathrm{pH}_{2}$ has been measured in [1] by means of Raman scattering spectra for rotational and vibrational transitions. The corresponding experimental data are shown in Fig. 2 (circles) jointly with our theoretical results (solid line).

Here and below we consider a constant flow rate through the filament. In fact, estimates show that at the beginning of the filament for $z=0.02 \mathrm{~mm}, T=16 \mathrm{~K}$ (see Fig. 2), the value of the evaporation mass flow $d Q_{2} /\left(c_{2} T\right)$ according to Eq. (2), accounting for the exponential factor from Eq. (3), is about $10 \%$ of the main mass flow $d Q_{1} /\left(c_{1} d T\right)$, see Eq. (1). The same estimate of $10 \%$ applies near the end of the filament $(z=0.9 \mathrm{~mm}, T=11.5 \mathrm{~K})$. This result is also in agreement with the experimental data [1] where a constant filament diameter is clearly seen.

In spite of the simplicity of our model the agreement with experiment [1] is fairly good. The small difference about $10 \%$ in the value of the temperature $T$ at the middle of the filament may be attributed to small amount of crystals at $T<T_{m}=13.8 \mathrm{~K}$ (freezing temperature for $\mathrm{pH}_{2}$ ). These small crystals in the surface of the filament give rise to an effective decrease of the evaporation rate causing an increase of the temperature. 


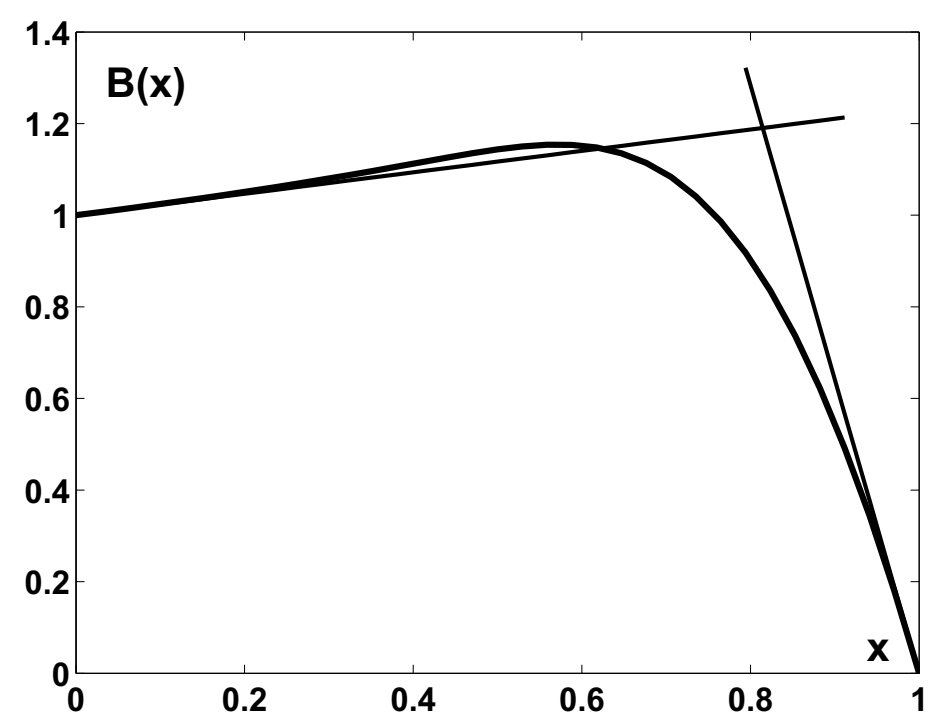

FIGURE 1. Calculated profile $\mathrm{B}(\mathrm{x})$, where $x=T / T_{0}$. Two asymptotes are shown.

\section{FILAMENT VELOCITY PROFILE}

We get from Eqs. (1) and (2) the exact expression for the temperature gradient

$$
\frac{\partial T}{\partial z}=\frac{5 R}{D u_{0} A_{2}} \sqrt{\frac{\pi k T}{m}} \exp \left(-\frac{q}{R T}\right)
$$

which enables studying the velocity profile inside the filament due to the surface-tension effects [8].

Considering a steady-state liquid filament of length $L$ and diameter $D=2 R_{0}$ at $L>>D$ in $(r, z)$ geometry, the solution of the Navier-Stokes equation system describing the flow in the filament has the form

$$
u_{z}=u(r), \quad u_{r}=0, \quad P=P(z),
$$

where $u_{r}$ and $u_{z}$ are the radial and the axial velocities, respectively, and $P$ is the pressure.

The corresponding Navier-Stokes equations for incompressible flow have the form

$$
\frac{d P}{d z}=\mu \frac{1}{r} \frac{d}{d r}\left(r \frac{d u}{d r}\right), \quad \frac{d P}{d z}=\text { const }
$$

where $\mu=18.485 \cdot 10^{-6} \mathrm{~Pa} \cdot \mathrm{s}$ is the viscosity coefficient.

The Marangoni boundary conditions at $r=R_{0}$ imply the equality of the thermocapillary surface-tension forces $F_{t}$ and the shear-stress ones:

$$
\mu \frac{d u}{d r}=F_{t}=\frac{\partial \sigma}{\partial z}=\frac{\partial \sigma}{\partial T} \frac{\partial T}{d z}
$$

where $\sigma(T)$ is the surface tension coefficient. A positive value of $F_{t}>0$ results from $\partial \sigma / \partial T<0$, and $\partial T / \partial z<0$.

The system (14) is solved for the boundary condition (15) at $r=R_{0}$. The additional constraint for radial velocity $u(r)$ at the filament axis $r \rightarrow 0$, together with the constant flow rate along the filament leads to

$$
2 \pi \int_{0}^{R_{0}} u_{0} r d r=\pi R_{0}^{2} u_{0}=2 \pi \int_{0}^{R_{0}} u r d r
$$

By integration one obtains the velocity profile 


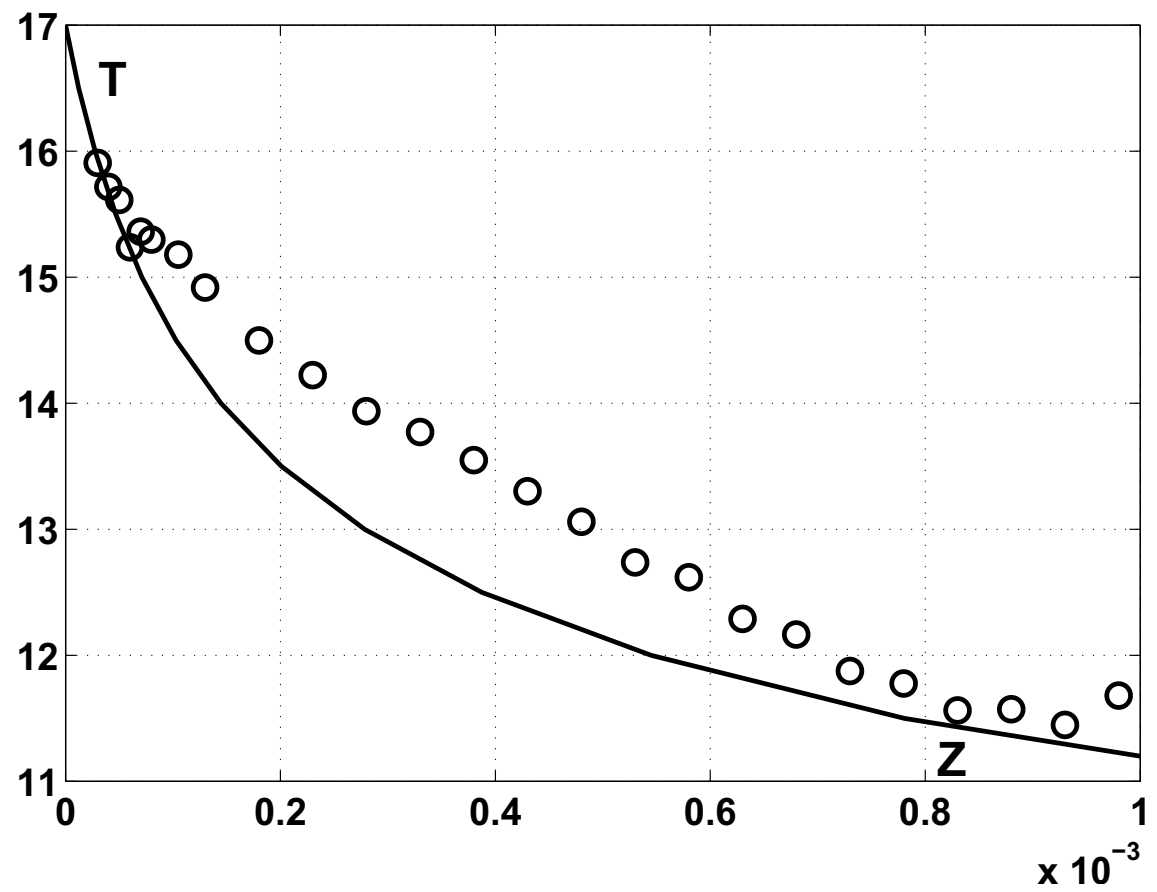

FIGURE 2. Experimental (circles, from [1]) and calculated (full line) temperature-distance profiles of a $D=4 \mu \mathrm{m}$ undercooled filament of liquid $\mathrm{pH}_{2}$. Parameters: nozzle temperature $T_{0}=17 \mathrm{~K}$, flow velocity $u_{0}=200 \mathrm{~ms}^{-1}$, from $[1] ; A_{2}=0.932 \mathrm{~J}$ mol ${ }^{-1}$ $\mathrm{K}^{-2}$, from [6]; evaporation heat $q=904 \mathrm{~J} \mathrm{~mol}^{-1}$, from [5]; $T_{1}=109 \mathrm{~K} ; L_{0}=0.391 ; R=8.3145 \mathrm{~J} \mathrm{~K}^{-1} \mathrm{~mol}^{-1}$, the universal gas constant; $m=3.2 \cdot 10^{-27} \mathrm{~kg}$, mass of $\mathrm{pH}_{2}$ molecule.

$$
u(r)=u_{0}+\frac{\alpha}{2 R_{0}^{2}}\left(r^{2}-\frac{1}{2} R_{0}^{2}\right)
$$

where

$$
\alpha=R_{0} \frac{1}{\mu} \frac{\partial \sigma}{\partial T} \frac{\partial T}{\partial z}>0
$$

The profile obtained in (17) is a parabola with

$$
u(0)=u_{0}-\frac{\alpha}{4}, \quad u\left(R_{0}\right)=u_{0}+\frac{\alpha}{4},
$$

which can be unstable. A scheme of the velocity distribution is shown in Fig. 3.

The pressure gradient due to the Marangoni forces is positive

$$
\frac{d P}{d z}=\frac{2 \mu \alpha}{R_{0}^{2}}=\frac{2}{R_{0}} \frac{\partial \sigma}{\partial T} \frac{\partial T}{\partial z}>0,
$$

and the additional positive pressure is

$$
P(z)=\int_{0}^{L} \frac{d P}{d z}=\frac{2 \sigma}{R_{0}} .
$$

This value is equal to the additional pressure under the curve surface due to the surface tension, see e.g. [5] and [8].

We use the approximate formula for the surface tension coefficient from [8], which is valid for a large number of liquids,

$$
\sigma(T)=B\left(T_{0}-T-\tau\right)\left(\frac{\rho_{0}}{\mu_{m}}\right)^{2 / 3}
$$


where $B=2.1$ in CGS system, $T_{0}=17 \mathrm{~K}$ is the critical temperature, $\tau$ is a small constant temperature, and $\mu_{m}$ is the molecular weight of the liquid. From Eq. (20) one obtains

$$
\frac{\partial \sigma}{\partial T}=-B\left(\frac{\rho_{0}}{\mu_{m}}\right)^{2 / 3}=-0.265 \cdot 10^{-3} \frac{\mathrm{N}}{\mathrm{m} \cdot \mathrm{K}} .
$$

Using (21) and the temperature gradient (12) we calculate from Eq. (18) the $\alpha$ value for the velocity profile (17). Since the temperature gradient along the filament changes strongly, we report the estimate $\alpha \approx 0.10 \mathrm{~m} \mathrm{~s}^{-1}$ at $z=500 \mu \mathrm{m}$. For $z>1000 \mu \mathrm{m}$ the filament crystallizes into a solid [1].

As a result of the effect of surface tension coefficient $\partial \sigma / \partial T$, the velocity profile across the filament

$$
u(r)=u_{0}+2 C\left(r^{2}-0.125 D^{2}\right) / D^{2},
$$

is obtained, where $C \approx 0.1 \mathrm{~m} \mathrm{~s}^{-1}$ at $z=500 \mu \mathrm{m}$. Although this disturbance is small, it might induce crystal growth habits differing from those of bulk solid $\mathrm{pH}_{2}$.
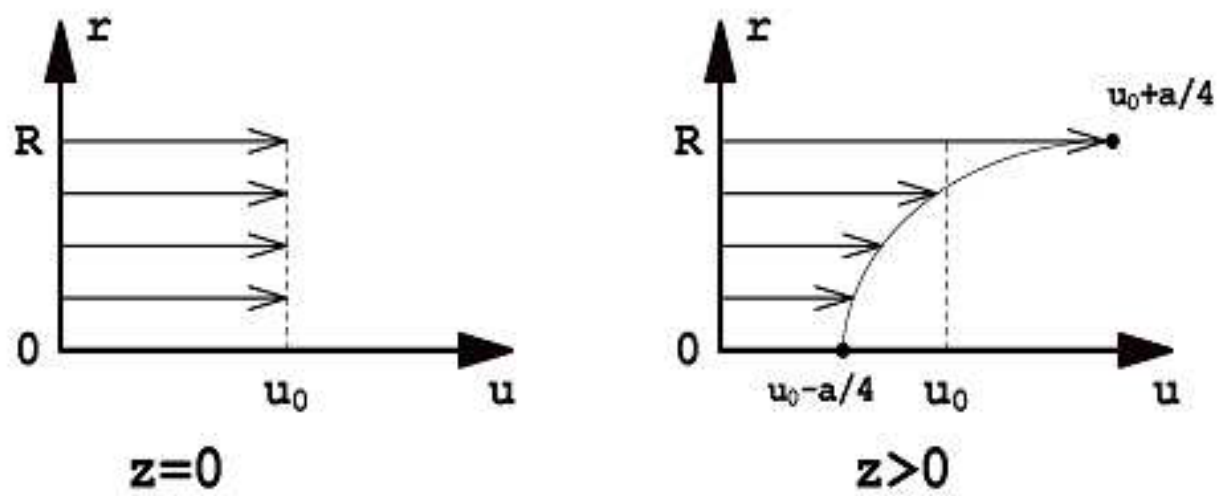

FIGURE 3. Scheme of the velocity distribution $u(r)$ along the filament at the nozzle exit (left) and in the some middle point (right)

\section{CONCLUSIONS}

In the present paper a simple model for the heat flow balance between a liquid medium and a rarefied gas environment allows predicting with fairly good accuracy the experimental [1] temperature distribution along an undercooled filament of liquid $\mathrm{pH}_{2}$.

With aid of the main thermal and viscosity parameters a parabolic velocity profile across the jet is inferred which might have consequences for the cristallization process going on downstream in the the filament.

The transverse distribution of the temperature inside the filament has also been observed [1]. In our simple analytical model this distribution is not considered so far, but it may be the subject of future theoretical work.

\section{REFERENCES}

1. M. Kühnel, J. M. Fernández, G. Tejeda, S. Montero, and R. E. Grisenti, Phys. Rev. Lett. 106, 245301-1-4 (2011).

2. V. L. Ginzburg, A.A. Sobyanin, JETP Lett. 15, 242 - 244 (1972).

3. S. Grebenev, B. Sartakov, J. P. Toennies, and A. Vilesov, Science 289, 1532 - 1535 (2000).

4. H. Li, R. J. Le Roy, P. N. Roy, and A. R. W. McKellar, Phys. Rev. Lett. 105, 133401-1-4 (2010). 
5. D. V. Sivoukhin, Molecular Physics, Nauka, Moscow (1983)

6. H. J. Maris, G. M. Seidel,, and T. Huber, J. Low Temp. Phys. 51, 471-487 (1983)

7. J. L. Tell and H. J. Maris, Phys. Rev. B 28, 5122 - 5125 (1983)

8. A. K. Kikoin and I. K. Kikoin, Molecular Physics, Nauka, Moscow (1976). 